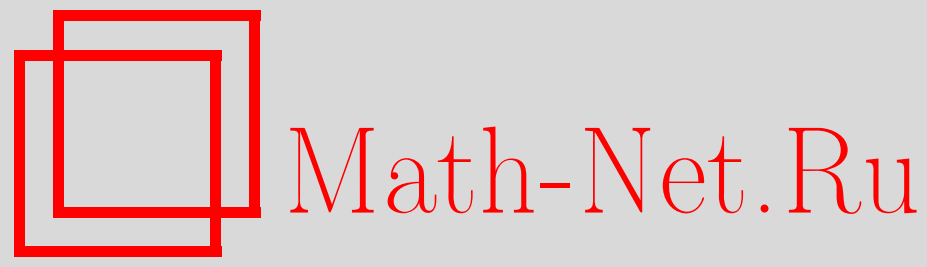

А. Ю. Фишкин, Формальная и аналитическая линеаризуемость семейств ростков конформных отображений $(\mathbb{C}, 0)$ в $(\mathbb{C}, 0)$, Функи. анализ и его прил., 2009, том 43, выпуск 1, 94-96

DOI: https://doi.org/10.4213/faa2945

Использование Общероссийского математического портала MathNet.Ru подразумевает, что вы прочитали и согласны с пользовательским соглашением

http://www . mathnet.ru/rus/agreement

Параметры загрузки:

IP : 54.224 .135 .184

26 апреля 2023 г., 11:49:33

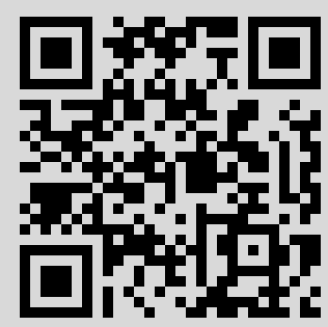




\title{
Формальная и аналитическая линеаризуемость семейств ростков конформных отображений $(\mathbb{C}, 0)$ в $(\mathbb{C}, 0)$
}

\author{
(c) 2009. А. Ю. Фишкин*
}

Рассмотрим росток конформного отображения $F(z):(\mathbb{C}, 0) \rightarrow(\mathbb{C}, 0)$ с неподвижной точкой 0 и мультипликатором $\lambda=F^{\prime}(0) \neq 0$. При $|\lambda| \neq 1$ такой росток аналитически эквивалентен своей линейной части в нуле (теорема ШрёдераКёнигса [1, с. 31]). При $|\lambda|=1$ ответ на вопрос об аналитической эквивалентности заданного ростка линейному напрямую связан с арифметическими свойствами числа $\lambda$.

Пусть $\lambda=e^{2 \pi i \varepsilon}$, причем число $\varepsilon \in \mathbb{R}$ диофантово, т. е. найдутся $C>0$ и $\sigma>2$, такие, что для любого $p / q \in \mathbb{Q}$ справедливо неравенство

$$
|\varepsilon q-p|>C / q^{\sigma} \text {. }
$$

Для произвольных $C$ и $\sigma$ это свойство выполняется для почти всех вещественных $\varepsilon$. Приведем теорему Зигеля, несколько усиленную по сравнению с традиционной формулировкой [1, с. 43]:

Теорема Зигеля. Пусть $F(z)=\lambda z+O\left(z^{2}\right)$ - росток голоморфной функиии в нуле $и \varepsilon=\frac{1}{2 \pi i} \log \lambda \in \mathbb{C}$ удовлетворяет условию (1) для заданных $C>0$, $\sigma>2$ и всех $p / q \in \mathbb{Q}$. Тогда найдется положительное $\delta=\delta(C, \sigma)$, такое, что если для некоторого $r>0$ в диске $|z|<2 r$ выполняется неравенство $\left|F^{\prime}(z)-\lambda\right|<\delta$, то в диске $|z|<r$ отображение $F(z)$ аналитически сопряжено линейному отображению $\lambda z$, причем для некоторого $M(r, C, \sigma)$ сопрягающее отображсение $H(z)$ удовлетворяет условию

$$
\max _{|z| \leqslant r}|H(z)|<M(r, C, \sigma) .
$$

Замечание. Приведенная формулировка теоремы Зигеля содержит два обобщения по сравнению с классической. Во-первых, не требуется, чтобы $\varepsilon$ было вещественным (т. е. не обязательно, чтобы мультипликатор $\lambda$ принадлежал единичной окружности в $\mathbb{C}$ ). Во-вторых, даны оценки на максимум модуля сопрягающего отображения и на размер диска, в котором оно определено. Оценка на максимум не зависит от конкретного $\varepsilon$, она зависит лишь от параметров $C$ и $\sigma$, фигурирующих в условии (1). Справедливость этих обобщений непосредственно следует из доказательства теоремы Зигеля, изложенного в [1].

Перейдем к рассмотрению ростков аналитических семейств конформных отображений с неподвижной точкой 0 :

$$
F_{\varepsilon}(z):\left(\mathbb{C}^{2}, 0\right) \rightarrow\left(\mathbb{C}^{2}, 0\right), \quad(\varepsilon, z) \in \mathbb{C}^{2}, \quad F_{0}(z)=\lambda z+O\left(z^{2}\right) .
$$

Если $0<|\lambda| \neq 1$, то для таких локальных семейств имеет место аналог теоремы Шрёдера-Кёнигса: существует росток $H_{\varepsilon}(z)$ (аналитический по $\varepsilon$ и $z$ ), сопрягающий $F_{\varepsilon}(z)$ с линейным семейством $R_{\varepsilon}(z)=F_{\varepsilon}^{\prime}(0) z$.

\footnotetext{
* Работа частично поддержана грантами РФФИ 7-01-00017-а и 05-01-02801-CNRS_a.
} 
Пусть теперь $|\lambda|=1$. Назовем семейство (2) невырожденным, если отображение $\varepsilon \rightarrow F_{\varepsilon}^{\prime}(0)$ конформно при $\varepsilon=0$. Тогда, производя аналитическую замену параметра, можно считать, что

$$
F_{\varepsilon}(z)=e^{2 \pi i \varepsilon} z+O\left(z^{2}\right), \quad \varepsilon \in\left(\mathbb{C}, \varepsilon_{0}\right),
$$

где $\varepsilon_{0}=\frac{1}{2 \pi i} \log \lambda \in \mathbb{R}$. По теореме Пуанкаре-Дюлака семейство $F_{\varepsilon}(z)$ можно упростить с помощью формального сопряжения $\widehat{H}_{\varepsilon}(z)=z+\sum_{k>1} h_{k}(\varepsilon) z$. Это значит, что мы не требуем сходимости ряда $\widehat{H}_{\varepsilon}(z)$, однако при сопряжении с помощью этого ряда $F_{\varepsilon}(z)$ приводится к формальной нормальной форме Пуанкаре-Дюлака $\widehat{F}_{\varepsilon}(z)$, где

$$
\widehat{F}_{\varepsilon}(z)= \begin{cases}e^{2 \pi i \varepsilon} z & \text { для } \varepsilon \in \mathbb{Q}, \\ e^{2 \pi i \varepsilon} z+\sum_{k>0} c_{k q+1}(\varepsilon) z^{k q+1} & \text { для } \varepsilon=\frac{p}{q} \in \mathbb{Q} \text { с взаимно простыми } p \text { и } q,\end{cases}
$$

причем коэффициенты $c_{k q+1}(\varepsilon)$, вообще говоря, отличны от 0. Очевидно, что для того, чтобы локальное семейство было аналитически сопряжено линейному, необходимо, чтобы его формальная нормальная форма была линейной для всех $\varepsilon$. Мы докажем теорему, утверждающую, что это условие является также и достаточным.

Теорема. Пусть $\varepsilon_{0} \in \mathbb{R}$ и локальное семейство (3) формально эквивалентно линейному в достаточно малом диске $\left|\varepsilon-\varepsilon_{0}\right|<\rho$. Тогда это семейство аналитически эквивалентно линейному, а именно, существует росток аналитической замены координат $H_{\varepsilon}(z)$ в точке $(\varepsilon, z)=\left(\varepsilon_{0}, 0\right)$, для которого

$$
H_{\varepsilon}^{-1}(z) \circ F_{\varepsilon}(z) \circ H_{\varepsilon}(z)=e^{2 \pi i \varepsilon} z .
$$

Лемма. Пусть $\varepsilon_{0} \in \mathbb{R}$ и локалъное семейство (3) формально эквивалентно линейному в достаточно малом диске $\left|\varepsilon-\varepsilon_{0}\right|<\rho$. Тогда коэффиииенты $h_{k}(\varepsilon)$ формальной линеаризующей замень $\widehat{H}_{\varepsilon}(z)=z+\sum_{k>1} h_{k}(\varepsilon) z^{k}$ голоморфнь в этом диске.

Доказательство. Пусть $T_{\varepsilon}(z)=e^{2 \pi i \varepsilon} z+\sum_{k>1} f_{k}(\varepsilon) z^{k}-$ ряд Тейлора для функции $F_{\varepsilon}(z)$ в точке $z=0$ при $\left|\varepsilon-\varepsilon_{0}\right|<\rho$, а $\widehat{H}_{\varepsilon}(z)-$ ряд, выписанный выше. По определению формальной линеаризующей замены для этих рядов справедливо тождество

$$
T_{\varepsilon}(z) \circ \widehat{H}_{\varepsilon}(z)=\widehat{H}_{\varepsilon}\left(e^{2 \pi i \varepsilon} z\right),
$$

из которого приравниванием коэффициентов при одинаковых степенях переменной z получаются рекуррентные соотношения на функции $h_{k}(\varepsilon)$ вида

$$
h_{k}(\varepsilon) \cdot\left(e^{2 \pi i k \varepsilon}-e^{2 \pi i \varepsilon}\right)=A_{k}\left(h_{2}, h_{3}, \ldots, h_{k-1}, f_{2}, \ldots, f_{k}\right),
$$

где $A_{k}$ - некоторые многочлены от своих аргументов. Отсюда следует, что если функции $h_{2}(\varepsilon), \ldots, h_{k-1}(\varepsilon)$ аналитичны, то $h_{k}(\varepsilon)$ может иметь при $\left|\varepsilon-\varepsilon_{0}\right|<\rho$ лишь конечное число особенностей в точках вида $\varepsilon_{j}=j /(k-1)$. Поскольку семейство формально приводимо к семейству поворотов при $\left|\varepsilon-\varepsilon_{0}\right|<\rho$, то значение $h_{k}\left(\varepsilon_{j}\right)$ определено и, тем самым, все эти особенности устранимы. Следовательно, функция $h_{k}(\varepsilon)$ голоморфна. Доказательство завершается применением математической индукции. 
Доказательство теоремы. Возьмем достаточно близкие к $\varepsilon_{0}$ диофантовы числа $\varepsilon_{1}, \varepsilon_{2}$, удовлетворяющие соотношению (1) с некоторыми параметрами $C$, $\sigma$ и такие, что $\varepsilon_{1}<\varepsilon_{0}<\varepsilon_{2}$. Рассмотрим прямоугольник $K \subset \mathbb{C}$ со сторонами $\operatorname{Re} z=\varepsilon_{1}, \operatorname{Re} z=\varepsilon_{2}, \operatorname{Im} z=\delta, \operatorname{Im} z=-\delta$, где $\delta$ настолько мало, что $K$ компактно содержится в диске $\left|\varepsilon-\varepsilon_{0}\right|<\rho$. Заметим, что для $C^{\prime}=\min (C, \delta), \varepsilon \in \partial K$ и $q / p \in \mathbb{Q}$ справедливо неравенство

$$
|\varepsilon q-p|>C^{\prime} / q^{\sigma} .
$$

Действительно, для $\varepsilon$ на вертикальных сторонах прямоугольника $K$

$$
|\varepsilon q-p| \geqslant|q \operatorname{Re} \varepsilon-p|=\left|q a_{i}-p\right|>C / q^{\sigma},
$$

а на его горизонтальных сторонах

$$
|\varepsilon q-p| \geqslant|\operatorname{Im} \varepsilon|=\delta>\delta / q^{\sigma} .
$$

Применяя теорему Зигеля в приведенной выше формулировке, мы получим, что для $\varepsilon \in \partial K$ определено аналитическое сопряжение $H_{\varepsilon}(z)$ ростка $F_{\varepsilon}$ с линейным отображением и, кроме того, $\left|H_{\varepsilon}(z)\right|<M$ для $|z|<r$ при некоторых $M$ и $r$, зависящих от $F_{\varepsilon}, C, \delta$ и $\sigma$. Поскольку ряд Тейлора для $H_{\varepsilon}(z)$ совпадает с формальным рядом $\widehat{H}_{\varepsilon}(z)$, коэффициенты которого равны $h_{k}(\varepsilon)$, то в силу неравенств Коши для $\varepsilon \in \partial K$ выполнено неравенство

$$
\left|h_{k}(\varepsilon)\right|<M / r^{k} .
$$

Учитывая, что функции $h_{k}(\varepsilon)$ голоморфны в $\bar{K}$ в силу доказанной леммы, получим, что та же оценка на $\left|h_{k}(\varepsilon)\right|$ верна и для $\varepsilon \in K$ по принципу максимума. Отсюда легко заключить, что для $\varepsilon \in K$ ряд $\widehat{H}_{\varepsilon}(z)$ сходится равномерно на компактах в круге $|z|<r$ к аналитической линеаризующей замене $H_{\varepsilon}(z)$. Голоморфность $H_{\varepsilon}(z)$ по $\varepsilon$ внутри $K$ при фиксированном $z,|z|<r$, следует из теоремы Вейерштрасса о равномерной сходимости, а голоморфность по обоим аргументам $\varepsilon, z-$ из теоремы Хартогса.

Автор сердечно благодарен Ю. С. Ильяшенко за ценные замечания и внимание к этой работе.

\section{ЛитератУРА}

[1] L. Carleson, T. W. Gamelin, Complex Dynamics, Springer-Verlag, New York, 1993.

Независимый московский университет

Поступило в редакцию 25 сентября 2007 г.

Заведующая редакцией и научный редактор Г. М. Цукерман

Сдано в набор 19.12.2008. Подписано к печати 26.01.2009. Формат 70×100/16

Печать офсетная. Усл. печ. л. 7,8. Усл. кр.-отт. 2,1 тыс. Бум. л. 3,0 Уч.-изд. л. 9,0. Тираж 257 экз. Заказ 903.

Учредитель: Российская академия наук

Адрес редакции: 117966 Москва, ГСП-1, ул. Губкина 8, комн. 624. Тел. 938-37-56 Издатель: Российская академия наук, Издательство «Наука», 